\title{
LA CONSOLIDACIÓN DE LA OFICINA MUNICIPAL DE LA MUJER. EL CASO DE HEREDIA
}

\section{THE CONSOLIDATION OF MUNICIPAL OFFICE FOR WOMEN. THE CASE OF HEREDIA}

Meyling González Espinoza*

RESUMEN

El artículo presenta la experiencia en la gestión de políticas y acciones que promuevan la igualdad y la equidad de género en el ámbito de la Municipalidad de Heredia, Costa Rica. Se analiza el proceso de gestión estratégica que muestra la complementación con los avances globales y nacionales en esta temática en el ámbito municipal. En muchos sentidos, es una guía para el trabajo de promoción en un ámbito donde estas políticas y acciones requieren de intervenciones y prácticas de excelencia, por su escaso desarrollo en el país.

PALABRAS CLAVE: MUJERES * ESTRATEGIA DE GESTIÓN * IGUALDAD DE GÉNERO * POLÍTICA GUBERNAMENTAL * ALIANZA

\section{ABSTRACT}

The article presents the experience of management policies and actions to promote gender equality and equity in the area of the Municipality of Heredia. It's analyzed the strategic management process that shows the complementation with global and national developments in this topic at the municipal level. It's a guide to the work of promoting in an area where these policies and actions required interventions and practices of excellence for their poor development in the country.

KEYWORDS: WOMEN * MANAGEMENT STRATEGY * GENDER EQUALITY * GOVERNMENT POLICY * ALLIANCES

Escuela de Planificación Económica y Promoción Social de la Universidad Nacional (Costa Rica). meygoes88@gmail.com 
UNA EXPERIENCIA DE GESTIÓN EMBLEMÁTICA QUE MARCA CAMINO EN LA INSTITUCIONALIDAD PÚBLICA

A 15 años de su apertura y 10 años de realizar una gestión con visión estratégica, la Oficina Municipal de la Mujer (оғIм) de Heredia logra - a pesar de situaciones adversas (por ejemplo, ubicarse bajo las gradas del Palacio Municipal)_ posicionarse como uno de los órganos consolidados a nivel local en lo que se refiere a la promoción, protección y defensa de los derechos de las mujeres con respaldo comunal, institucional y político.

Este proceso demuestra la experiencia del Cantón Central de Heredia, la que fue considerada por el Instituto Nacional de las Mujeres como emblemática y avanzada, por ser de las pocas experiencias que goza de presupuesto propio, cuenta con respaldo político y trabaja con visión social y de género (Vargas, 2013).

Estas atribuciones son producto de la gestión desarrollada desde y por la ofim de Heredia, que al combinar elementos estratégicotácticos, la han llevado a ser vanguardia en materia de políticas públicas de género, dentro de un marco adverso conocido como Estado "garantista" (ofrece mucho y entrega poco).

El éxito de la gestión estratégica se cimenta en un proceso que concita apoyos locales, nacionales e internacionales. El involucramiento de actores le confirió crecimiento, progreso y respaldo, con el que logra abrir un espacio institucional operativo y da respuesta a las necesidades de la población de su cantón.

Sus logros y aportes a la comunidad marcan camino en la planificación y gestión pública del país, al demostrar la posibilidad de llevar a cabo procesos, mediante la construcción de estrategias basadas en el involucramiento y la construcción de capital social.

\section{PROBLEMAS INSTITUCIONALES Y POLÍTICOS PARA EJECUTAR POLÍTICAS PÚBLICAS}

La estrategia de gestión desarrollada por la оғIм de Heredia se inscribe en un marco institucional adverso, el análisis del proceso y los resultados de la estrategia que se presentan, señalan elementos que influyen y participan de diversas formas en la institucionalidad pública y explican en parte - sin ser determinantes absolutos- el entrabamiento institucional del país.

Entre estos elementos se encuentra la brecha entre la promesa democrática y la capacidad de hacer del Estado para entregar servicios y bienes de calidad, la carencia de sistemas de evaluación y control sobre resultados, la sobre normatividad proveniente de problemas institucionales de operatividad para ejecutar las políticas públicas y la incapacidad de adaptación institucional.

El Estado costarricense maneja una promesa democrática extremadamente grande, por medio de la aprobación de leyes, los ciudadanos tienen derecho a la salud, a la educación, a la protección social y así sucesivamente, más de 500 leyes se han encargado de ampliar la promesa democrática. Desde el año 90 a la fecha, el $80 \%$ de estas leyes comprometen recursos públicos; sin embargo, solo el $29 \%$ especifica de dónde provienen (Programa Estado de la Nación, 2013).

Esta situación da como resultado un Estado que promueve leyes, simplemente garantistas de derechos, ya que carecen de contenido presupuestario para su debida ejecución.

Además de carecer de capacidades necesarias para cumplir con sus responsabilidades, en Costa Rica prácticamente no existe un sistema de evaluación de políticas y servicios estatales (Straface y Vargas, 2008).

Pese a que cuenta con una serie de controles horizontales, que ejercen propiamente la Contraloría General de la República, la Defensoría de los Habitantes y la Procuraduría General de la República; la gestión administrativa resulta ineficiente, ante la falta de sistemas de evaluación sobre resultados.

Straface y Vargas, insisten que "la combinación de múltiples controles sin sistemas de evaluación y el debilitamiento de las bases fiscales de la acción pública, ha creado un serio problema estructural de gestión”(2008:123).

De esta manera, el control es ejercido sobre los procedimientos y no sobre los resultados y los procesos. Los controles de detalles $y$ de procedimientos, no solamente se alejan del control de resultados, también alejan a la ciudadanía 
de exigir responsabilidades a los funcionarios públicos como lo estipula el artículo 11 de la Constitución Política.

Por otra parte, una causa importante que paraliza a la administración pública es la sobrenormatividad, leyes, reglamentos e instancias de control que se convierten en mecanismos que lejos de construir una gestión pública eficiente, se convierten en herramientas administrativas que entraban $y$ hacen cada vez más ineficiente las instituciones.

Además de las condiciones desfavorables del marco institucional nacional, las oficinas municipales de la mujer enfrentan limitaciones en el contexto municipal, debido a que su origen externo y campo de trabajo, han tenido que operar en un marco de acción limitado.

Para el 2011, el Instituto Nacional de las Mujeres (гnamu), elaboró un perfil sobre los Mecanismos Locales para la Igualdad y Equidad de Género (melieg) ${ }^{1}$, en el que registró un total de 67 Oficinas, de las cuales 12 trabajan sin presupuesto propio y no se les asigna ninguna partida del presupuesto municipal, 9 llevan a cabo su programación anual con un presupuesto de un millón de colones o menos y 27 trabajan con un presupuesto que se ubica entre un millón y cinco millones de colones, 6 oficinas manejan un presupuesto de cinco a diez millones, 3 realizan su quehacer con un presupuesto de diez a quince millones y 2 de ellas cuentan con un presupuesto mayor a los quince millones, 3 operan con un presupuesto mayor a veinte millones y únicamente, la Oficina de San Carlos tiene un presupuesto de treinta millones de colones, no obstante, esta se encarga de todos los temas del Área de Desarrollo Social de la Municipalidad. La Oficina Municipal de la Mujer de Heredia, es una de las tres oficinas que cuenta con un presupuesto mayor a veinte millones de colones; para el 2011, la Oficina de este cantón operó un presupuesto de $₫ 25943255,00$.

1 Los organismos de género en las municipalidades, se concibieron bajo el nombre de ofIM; sin embargo, debido a los cambios de nombre que se han dado en las diferentes municipalidades $y$ a la diversidad de sus quehaceres, actualmente se denominan MELIEG.
Además de llevar a cabo sus funciones con recursos muy limitados, no se les reconoce en el organigrama institucional y las posibilidades de acción se encuentran sujetas a la disponibilidad de las autoridades políticas.

\section{LA OFIM DEL CANTÓN CENTRAL DE HEREDIA COMO CONSTRUCTORA DE SU GESTIÓN ESTRATÉGICA}

\section{¿ADMINISTRAR O GESTIONAR UNA OFIM?}

La ofımde Heredia inició sus labores en 1999, en un contexto de incomprensión institucional y sin respaldo político; esta situación la llevó a trabajar en un escenario de limitaciones y dificultades operativas. Así, la ofim de Heredia, al igual que los demás órganos de género de nivel local, realizó sus labores sin presupuesto propio, en un espacio no adecuado, sin reconocimiento en la estructura municipal, y sin una visión ni funciones claras. Consecuentemente, desarrolló su quehacer con carácter asistencial.

Sin embargo, a partir del 2004, esta oficina encarriló sus esfuerzos hacia la conformación de un nicho institucional, por medio de una estrategia de gestión guiada por principios estratégicos que han tenido desarrollo en el arte militar y político, destacándose la capacidad de visión estratégica de la ofim. Puesto que en ese momento implicaba no solo trascender las limitaciones institucionales mencionadas, sino también posicionarse como un órgano local de promoción, protección y defensa de los derechos de las mujeres.

Con esa perspectiva, la ofim se dio a la tarea de realizar un proceso basado en la gestión estratégica orientado a involucrar diversas instancias municipales de decisión y a un sector beneficiario de su labor, del Cantón Central de Heredia, en la identificación de necesidades y en la tarea de perfilar acciones $y$ procesos, lo cual ha aportado a su experiencia y le ha permitido avanzar en su legitimación institucional.

\section{LO ESTRATÉGICAMENTE DESEABLE Y LO TÁCTICAMENTE POSIBLE}

En un contexto donde las políticas públicas desde una visión de estrategia superior, 
resultan ineficientes, el surgimiento de una estrategia inferior desde el nivel regional y local, resulta trascendental para la concreción de metas nacionales.

De esta manera, la ofim del Cantón Central de Heredia desde su gestión estratégica ha realizado aportes y contribuciones hacia la concreción de metas nacionales en materia de género enmarcadas en la estrategia superior (la Política Nacional para la Igualdad y Equidad de Género-PIEG) elaborada desde el Estado.

A continuación se describen los avances $y$ contribuciones a la PIEg en el último decenio, desde la ofim del Cantón Central de Heredia, divididos por objetivo estratégico:

\section{CUIDO COMO RESPONSABILIDAD SOCIAL}

$\diamond \quad$ Apertura del Centro de cuido nocturno de niños y niñas en los Lagos de Heredia en el 2013, por medio de la articulación de fuerzas y participación de la Casa de la Cultura de Heredia y las madres interesadas.

\section{TRABAJO DE CALIDAD Y GENERACIÓN DE INGRESOS}

$\diamond \quad$ Capacitación y formación a más de 229 mujeres en el área de empresariedad, a través del programa "Creando empresarias".

\section{EDUCACIÓN Y SALUD DE CALIDAD A FAVOR DE LA IGUALDAD}

$\diamond \quad$ Realiza capacitaciones en materia de género con las personas funcionarias de la Municipalidad y con la comunidad, con el objetivo de sensibilizar y educar a la población del Cantón Central de Heredia.

$\diamond \quad$ Con el programa "Bebé, piénsalo bien", ha abordado el tema de embarazo adolescente y salud sexual con más de 1550 estudiantes de secundaria.

\section{PARTICIPACIÓN POLÍTICA Y DEMOCRACIA PARITARIA}

$\diamond \quad$ Conformación de la Red de Mujeres Heredianas en el 2012, con el objetivo de que se convierta en una auditoría ciudadana que controle y monitoree el cumplimiento de los compromisos adquiridos por el Municipio con la población femenina del Cantón.
PROTECCIÓN EFECTIVA DE LOS DERECHOS Y FRENTE A TODAS LAS FORMAS DE VIOLENCIA

$\diamond \quad$ Elaboración de un reglamento interno contra el hostigamiento sexual.

$\diamond \quad$ Ha atendido a más de 2556 mujeres por violencia intrafamiliar.

INSTITUCIONALIDAD ROBUSTA A FAVOR DE LA IGUALDAD Y LA PARTICIPACIÓN POLÍTICA

$\diamond \quad$ Aprobación de la Política Municipal de Igualdad y Equidad de Género, así como, su respectivo Plan Estratégico en el 2010.

$\diamond \quad$ Contar con un nicho institucional operativo.

\section{ASPECTOS DINAMIZADORES DE LA ESTRATEGIA DE} GESTIÓN

Debido a su carácter y su posición institucional, la ofim ha tenido que maniobrar de manera interna y externa de la Municipalidad, sumando continuamente aliados. Al mismo tiempo, ha reducido la desconfianza de quienes pasiva 0 activamente trataron de obstaculizar su gestión, mediante información y resultados positivos.

De esta manera, la ofim de Heredia logró ganar apoyo político y administrativo, así mismo, logró el respaldo de las organizaciones comunales $y$ de la sociedad civil, mediante los diversos programas y procesos emprendidos por la Oficina.

$\mathrm{El}$ involucramiento progresivo de la sociedad civil, la neutralización de opositores internos y la suma de aliados para la transversalización de las metas de género en instancias institucionales, han sido aspectos estratégico-tácticos dinamizadores para superar las condiciones socio-políticas que se presentaron durante el proceso de construcción de la estrategia de gestión.

\section{DE LA MARGINALIDAD INSTITUCIONAL A LA CONSOLIDACIÓN DE UN NICHO INSTITUCIONAL OPERATIVO}

FASES DEL PROCESO DE CONSTRUCCIÓN DE LA ESTRATEGIA DE LA OFIM DE HEREDIA

La construcción de la estrategia de gestión abarca un periodo que comprende los años 2004-2013. 
El desarrollo de la estrategia se presenta dividida en tres fases; la primera comprende el periodo de 2004-2006, que se describe como la fase de diagnóstico y puesta en marcha de proyectos; la segunda abarca el periodo de crisis y reorganización que se inició en el 2007-2010 y por último, la tercera fase, del 2011-2013, que se ha llamado fase de despliegue del fortalecimiento institucional $y$ comunal de la ofim.

\section{FASE 1: DIAGNÓSTICO Y PUESTA EN MARCHA}

La оғгм del cantón central de Heredia inició sus labores en 1999, tan solo a un año de establecida la política de género a nivel local. Durante los primeros años no contó con los recursos humanos apropiados y tuvo grandes limitaciones con los recursos financieros $y$ de mobiliario. A lo largo de este periodo, la oficina estuvo conformada por una persona, cuyo nombramiento era el de secretaria; no se dispuso de presupuesto propio y antes del 2003, se ubicó bajo las gradas del Palacio Municipal.

Para el 2004, se presentan cambios en la dirección de la ofim y por consiguiente, en su gestión. De esta manera, sin una visión y funciones claras, pero con el objetivo de mejorar la condición de las mujeres del cantón, así como, de cambiar la conceptualización de la oғiм como órgano asistencialista, la Oficina arranca con dos proyectos: Atención Psicoterapéutica en Violencia Intrafamiliar y Capacitación.

La atención psicoterapéutica operó como un proceso de diagnóstico, con su ejecución se identificó que las relaciones de género requerían más que la atención asistencial que se brindaba a las mujeres por violencia intrafamiliar.

En respuesta a las necesidades identificadas y con el antecedente de 356 mujeres reincidentemente víctimas de violencia doméstica por carecer de autonomía económica, la ofiм planteó para el 2005 dos nuevos proyectos, con el fin de abordar el desempleo del cantón y brindar a las mujeres herramientas: 1) capacitación empresarial y 2) intermediación laboral; este último promovido por el Ministerio de Trabajo, con quien se firmó el convenio para su puesta en marcha.

Por otra parte, se puso en evidencia que la atención individual era un proceso desgastante para la psicóloga encargada de la ofiм, debido a que el servicio recaía sobre una persona; por lo tanto, se decidió desconcentrar el trabajo de atención de violencia intrafamiliar. A partir de esta acción, surgieron dos modalidades para el 2005: 1) atención individual y 2) grupos de apoyo. Con la implementación de estas dos nuevas modalidades, se pretendía realizar un abordaje integral y especializado en violencia intrafamiliar.

Además de las modalidades que surgían de los procesos de atención de violencia intrafamiliar, la Alcaldía del periodo 2003-2007, presentó propuestas para que la ofim se convirtiera en el departamento encargado de atender proyectos sociales, lo cual fue asumido y adaptado a los recursos, conocimientos y objetivos de la ofim; de esta manera surgieron como dos nuevos proyectos impulsados por el Alcalde: "Bebé, piénsalo bien" para trabajar el tema de sexualidad y embarazo adolescente, y la sala de juegos para niños y niñas sobrevivientes de abuso e incesto.

En el 2006, en el proceso de ejecución de los proyectos, surgió una nueva iniciativa, el "Programa construyendo nuevas formas de vivir la masculinidad en Heredia". Esta acción nació como una propuesta realizada al Alcalde por parte de la Jueza del Juzgado de Violencia Doméstica de Heredia, la M.Sc. María Ester Brenes Villalobos $y$ de la encargada de la ofim. Para la puesta en marcha del grupo de terapia se contrató al Instituto wem.

La cantidad de programas aprobados por parte de las autoridades municipales de la administración 2003-2007, le proporcionó a la ofim presupuesto propio y le rindió posicionamiento institucional.

La introducción y ejecución de nuevos programas significó un involucramiento progresivo de la sociedad civil en la detección de necesidades, la suma de aliados externos para dar respuesta a estas necesidades de la población meta del Cantón Central de Heredia y un aumento en el personal de la ofim.

Al mismo tiempo que los programas se ejecutaban y se incrementaba la participación de diversos sectores de la población, se reducían los niveles de incomprensión sobre el quehacer de este órgano de género en la Municipalidad. 
Durante este primer periodo 2004-2006, la ofiм registró logros importantes tanto en su posicionamiento municipal, como en avances en el campo de la educación, la promoción y la defensa de los derechos de las mujeres del Cantón Central de Heredia.

\section{FASE 2: CRISIS, CONFLICTO Y REORGANIZACIÓN}

Para el año 2007, hubo una serie de acontecimientos que cambiaron la situación de la ofiм, situándola en una posición desfavorable.

Las situaciones que ubicaron a la оғIм en un contexto de inoperatividad $y$ estancamiento se relacionan con el cambio de alcalde, cierre y traslado de programas, recorte de presupuesto y rechazo de requisiciones, así como, traslado de personal.

De esta manera, la оғім presentó un retroceso en su gestión, que derivó de un clima institucional infértil por parte de las autoridades municipales; no obstante, las acciones estratégico-tácticas no se hicieron esperar y aunque no tuvieron efectos inmediatos, para el 2009 logran neutralizar y crear una aceptación pasiva por parte del Alcalde, lo que le permitió a la ofiм continuar con su proceso de legitimación y avance hacia un Cantón de igualdad y equidad.

Las acciones tácticas que respaldaron a la Oficina en esta coyuntura fueron: 1) la demanda impuesta por la encargada de la ofim ante la Defensoría de los Habitantes y el inamu, debido a una situación de acoso laboral por parte del Alcalde; 2) el apoyo de los aliados externos en la audiencia que dió el Concejo Municipal para exponer la situación de acoso laboral y 3) una llamada de atención de la Contraloría General de la República a la Municipalidad por registrar un superávit de la ofim en el presupuesto del 2007.

Este periodo de "crisis" significó un aprendizaje para la ofıм, que la llevó a ampliar sus horizontes de acción para contrarrestar sus debilidades. Para el 2010, retoma la perspectiva de construir $y$ legitimar un nicho institucional operativo, mediante el involucramiento y neutralización de actores internos de la Municipalidad.

De esta manera emprendió un proceso de acciones tácticas encadenadas $y$ articuladas hacia un objetivo específico: construir un nicho institucional operativo consolidado y legitimado.
En orden cronológico, estas acciones fueron la elaboración del Diagnóstico de Género, el cual evaluó la situación municipal en este campo; la formulación y puesta en marcha de la Política Municipal para la Igualdad y Equidad de Género con su respectivo plan estratégico, que consistió en un proceso participativo orientado bajo las líneas metodológicas de la Política Nacional de Igualdad y Equidad de Género (PIEG), el cual se abocó desde el momento de formulación, a involucrar y a sensibilizar a actores administrativos y políticos de la estructura municipal.

Ambos procesos involucraron tanto la participación de la sociedad civil como de aliados externos, dentro de los que destaca la colaboración de onU-HÁBITAT, así como, la participación progresiva de actores administrativos, que pasaron de ser fuerzas opositoras a apoyos activos como el Departamento de Planificación y el de Control Interno, en el proceso de construcción de un municipio de promoción de prácticas de igualdad y equidad.

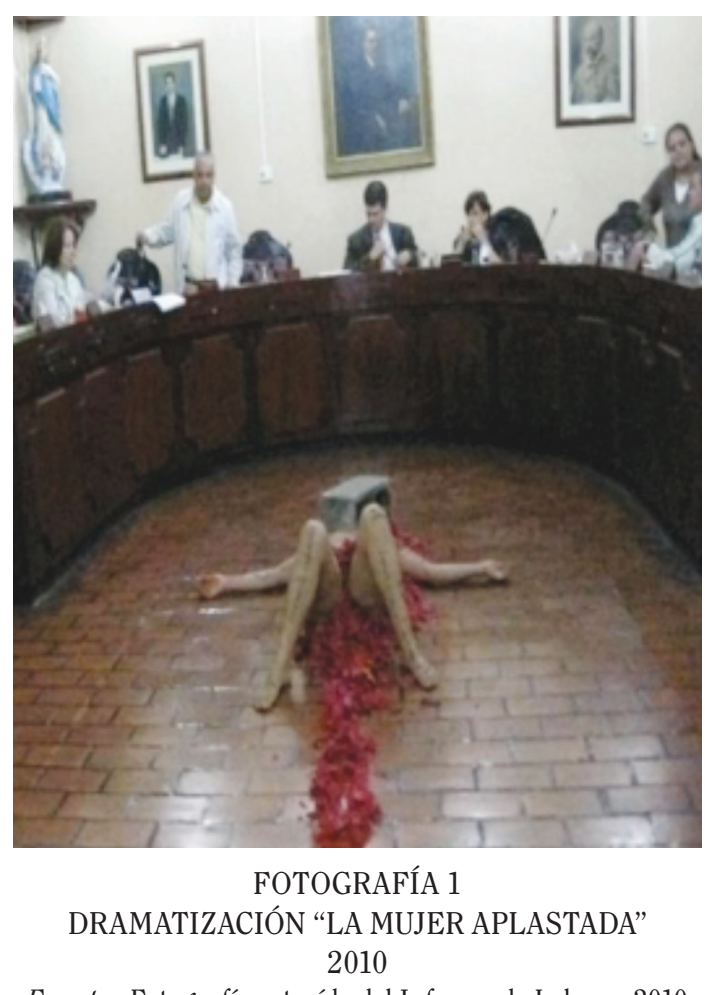

Fuente: Fotografía extraída del Informe de Labores 2010 de la ofIM de Heredia. 
En este periodo, la ofiм contó con el apoyo de actores administrativos, el respaldo de la Vicealcaldía y la Comisión de la Condición de la Mujer, que figuran como aliados políticos en el proceso.

Por otra parte, como un hito trascendental $y$ provocativo, la ofiм presenta sorpresivamente una dramatización "La mujer aplastada", en la que se plasmó la imagen de una mujer agredida y violada, al colocar a una mujer desnuda, con un bloque de cemento sobre la cara y con pétalos de flores rojas sobre su sexo que se prolongaban como un camino de sangre, en medio de la sala de sesiones del Concejo Municipal.

La acción generó disgusto, indignación y descontento por la "osadía" de la encargada de la ofim; sin embargo, este movimiento táctico, aunque chocante para los miembros del Concejo, cumplió con el objetivo de denuncia e información de las labores desempeñadas en esta oficina.

Posteriormente, en diciembre de 2010, la Comisión Municipal de la Condición de la Mujer presentó la Política y el Plan Estratégico ante el Concejo, la cual fue aprobada.

Durante el año 2010, la Oficina obtuvo dos principales logros: en primer lugar, la aprobación de la Política Municipal de Igualdad y Equidad de Género y, en segundo lugar, haber puesto en evidencia la importancia de su trabajo, tanto dentro de la Municipalidad como en la comunidad.

\section{FASE 3: DESPLIEGUE DEL FORTALECIMIENTO INSTITUCIONAL Y COMUNAL}

La ofim se dio a la tarea de fortalecer sus logros de la fase anterior, a través de un nicho institucional legitimado, por medio de la acumulación de fuerzas (con respaldo político, el de la Vicealcaldía; con un apoyo pasivo de actores internos neutralizados; con el apoyo activo de instituciones, organizaciones y grupos comunales como las asociaciones de desarrollo $y$ los grupos de emprendedoras), con un incremento sustancial en los recursos operativos y con líneas estratégicas definidas y plasmadas en un plan de acción.

De esta manera, la ofiм desplegó otras acciones tácticas y alcanzó resultados, con el objetivo de consolidarse como el órgano local encargado de promover los derechos de las mujeres y para afianzar el apoyo institucional, comunal y el respaldo político, así como, seguir aumentando fuerzas y reduciendo opositores.

Para el fortalecimiento institucional, con el apoyo activo del Departamento de Planificación, se incorporó como un objetivo del Plan Estratégico Municipal 2012-2016, el ejecutar la Política de Igualdad y Equidad de Género, y además se trazó como lineamiento que en los planes operativos anuales se incluyeran acciones específicas derivadas de esta Política. Por su parte, el apoyo por parte de Control Interno fue el de incluir la variable género dentro de su cuestionario de autoevaluación.

Para reducir las fuerzas opositoras internas y neutralizarlas, la táctica que se puso en práctica fue la de realizar talleres y cursos de capacitación, mediante los cuales se ha formado a la mayoría de los y las funcionarias. Estas actividades han servido a la vez como proceso de diagnóstico, ya que por medio de ellos se han identificado necesidades que están siendo tomadas como insumos para la elaboración de nuevos proyectos y para el mejoramiento de los actuales.

En cuanto al fortalecimiento comunal, se marcó con una acción estratégica, la de formar una Red de Mujeres Heredianas. Para esto, la táctica de la ofim consistió en organizar en el 2012, el Primer Encuentro de Mujeres organizadas de Heredia. Como parte del proceso de seguimiento en el 2013, se realizó el segundo encuentro. Allí se cumplió con el objetivo de reunir a la Red que se había conformado en el 2012 , a fin de que las mujeres expusieran ante las autoridades municipales sus necesidades e intereses, entre los que destacan su necesidad de contar con un espacio para comercializar sus productos.

Además, en este segundo encuentro se hizo perceptible el empoderamiento del grupo, cuya consolidación como una auditoría ciudadana se tiene como objetivo a mediano plazo, para que controle $y$ monitoree el cumplimiento de los compromisos adquiridos por el $\mathrm{Mu}-$ nicipio con la población femenina del Cantón Central de Heredia. 
Mediante la articulación de fuerzas, ejecuta proyectos interinstitucionales asumiendo retos e involucrando aliados en iniciativas de las que otras instituciones no se han querido hacer cargo, como el caso del centro de cuido nocturno de niños y niñas en los Lagos de Heredia. Este asunto le correspondía al Patronato Nacional de la Infancia (PanI); sin embargo, quienes asumieron el proyecto fueron la ofim y la Casa de la Cultura de Heredia. Asimismo, contó con la participación activa de las madres interesadas en que se abriera el centro, con el fin de poder asistir al colegio nocturno.

Entre algunos hechos importantes, se encuentra el reconocimiento internacional, de onu-Habitat, que recibió la Municipalidad por impulsar la igualdad y la equidad de género, tal y como se hizo con la Política de Género. Este acontecimiento hizo que el Alcalde se disculpara públicamente con la ofiм y su encargada por las situaciones presentadas en el 2007 y 2008.

Para el 2013, la Oficina Municipal de la Mujer desarrolla su planificación anual en concordancia con el Plan Estratégico de la Política, en el que se plasman seis proyectos:

1) Atención individual por violencia doméstica

2) Grupos de apoyo dirigido a mujeres

3) Construyendo nuevas masculinidades
4) Creando empresarias

5) Bebé, piénsalo bien

6) Colaboración con el programa de intermediación laboral y actividades paralelas para incorporar la perspectiva de género en la Municipalidad, así como, acciones afirmativas como conmemorar el Día a favor de los derechos de las mujeres.

En cuanto al presupuesto, este tuvo un crecimiento constante en comparación con periodos anteriores, al llegar a $\$ 69088$ 283,00, con una proyección para el 2014 de $\$ 120000000,00$.

\section{BALANCE DEL PROCESO DE GESTIÓN}

A manera de síntesis, se expone la situación de partida, de quiebre y de despliegue del proceso, donde se presenta el orden de los acontecimientos tomando en cuenta variables como presupuesto operativo de la ofim, personal, proyectos, colaboradores voluntarios y por contratación externa para la ejecución de los proyectos, así como, los aliados internos y externos que a su vez, se dividen en políticos y administrativos, organizaciones, instituciones y grupos comunales, $y$ las fuerzas opositoras. Este balance general permite ver los avances y los retrocesos en la gestión de la oғıм de Heredia. 
TABLA 1

BALANCE DEL PROCESO: ARRANQUE, CRISIS Y DESPLIEGUE DEL PROCESO DE GESTIÓN 2004-2013

\begin{tabular}{|c|c|c|c|c|c|}
\hline \multicolumn{3}{|c|}{ PERIODO } & 2004 & 2007 & 2013 \\
\hline \multicolumn{3}{|c|}{ Recursos Propios } & 12000000,00 & 14330680,00 & 69088283,00 \\
\hline \multicolumn{3}{|c|}{ Personal operativo de la ofIM } & $\begin{array}{l}1 \text { Secretaria } \\
1 \text { Psicóloga }\end{array}$ & 1 Psicóloga & $\begin{array}{l}1 \text { Secretaria } \\
1 \text { Psicóloga }\end{array}$ \\
\hline \multicolumn{3}{|c|}{ Proyectos } & $\begin{array}{l}\text { 1. Atención } \\
\text { psicoterapéutica } \\
\text { 2. Capacitación }\end{array}$ & $\begin{array}{c}\text { 1. Creando } \\
\text { Empresarias } \\
\text { 2.Construyendo } \\
\text { nuevas } \\
\text { masculinidades } \\
\text { 3. Atención de } \\
\text { Violencia Integral }\end{array}$ & $\begin{array}{l}\text { 1.Programa de atención de } \\
\text { Violencia Integral } \\
\text { 2. Creando empresarias } \\
\text { 3. Bolsa de empleo } \\
\text { 4. Construyendo nuevas } \\
\text { masculinidades } \\
\text { 5. Bebé, piénsalo bien }\end{array}$ \\
\hline \multicolumn{3}{|c|}{$\begin{array}{l}\text { Contratación externa y voluntarios para la } \\
\text { ejecución de proyectos }\end{array}$} & $\mathrm{X}$ & $\begin{array}{l}\text { INA } \\
\text { WEM }\end{array}$ & $\begin{array}{c}\text { Contratación de Co- } \\
\text { os.terapeutas } \\
\text { INA } \\
\text { MTSS } \\
\text { WEM } \\
\text { Estudiantes de Psicología y } \\
\text { Orientación y Ciencias } \\
\text { Sociales de universidades } \\
\text { públicas y privadas } \\
\text { Contratación de compañías } \\
\text { de teatro y de Somos como } \\
\text { somos }\end{array}$ \\
\hline \multirow{3}{*}{ Aliados } & Internos & $\begin{array}{c}\text { Políticos/ } \\
\text { Administrativos }\end{array}$ & $\begin{array}{l}\text { Alcaldía } \\
\text { Concejo } \\
\text { Municipal }\end{array}$ & $\begin{array}{l}\text { Clima } \\
\text { institucional } \\
\text { infértil }\end{array}$ & $\begin{array}{c}\text { Vicealcaldesa } \\
\text { Regidoras } \\
\text { Comisión de la Mujer } \\
\text { Planificación } \\
\text { Control Interno } \\
\text { Bolsa de empleo }\end{array}$ \\
\hline & \multirow[t]{2}{*}{ Externos } & $\begin{array}{l}\text { Instituciones/ } \\
\text { organizaciones }\end{array}$ & MEIC-MTSS-INAMU & $\begin{array}{l}\text { Red de Violencia } \\
\text { INAMU } \\
\text { IEM } \\
\text { Jueza de la } \\
\text { Defensoría de los } \\
\text { Habitantes } \\
\text { Poder Judicial }\end{array}$ & $\begin{array}{c}\text { INAMU } \\
\text { ONU- HÁBITAT } \\
\text { MEIC } \\
\text { MTSS } \\
\text { INA } \\
\text { Red local de prevención y } \\
\text { atención de violencia } \\
\text { MEP } \\
\text { WEM } \\
\text { Voluntarios universitarios } \\
\text { Contratación externa }\end{array}$ \\
\hline & & $\begin{array}{c}\text { Grupos } \\
\text { comunales }\end{array}$ & $\mathrm{X}$ & Guías y Scouts & Red de Mujeres Heredianas \\
\hline \multicolumn{3}{|c|}{$\begin{array}{l}\text { Opositores / Neutralizados/ Apoyo pasivo } \\
y \text { activo }\end{array}$} & $\begin{array}{l}\text { Alcalde/apoyo } \\
\text { pasivo } \\
\text { Funcionarios/ } \\
\text { apoyo pasivo }\end{array}$ & $\begin{array}{l}\text { Alcalde/ opositor } \\
\text { Funcionarios/ } \\
\text { pasivos }\end{array}$ & $\begin{array}{c}\text { Alcalde/apoyo pasivo } \\
\text { Funcionarios/apoyo activo y } \\
\text { pasivo }\end{array}$ \\
\hline
\end{tabular}

Fuente: Elaboración propia con datos aportados por la Oficina Municipal de la Mujer del Cantón Central de Heredia, 2013. 


\section{CONCLUSIONES}

Con la descripción del proceso de gestión de la Oficina Municipal de la Mujer de Heredia se muestra que, independientemente de las difíciles condiciones prevalecientes en la administración pública, puede propiciarse un buen marco de acción, si se cuenta con una estrategia que involucra los intereses locales y a actores institucionales.

La ofim de Heredia con la construcción de su estrategia de gestión, ha logrado contribuir de manera efectiva a la concreción de metas de la estrategia superior, al crear un nicho consolidado en el ámbito institucional municipal para el cumplimiento de la Política Nacional de Igualdad y Equidad de Género.

Se logró observar durante el artículo que el éxito de la estrategia de la ofim responde a acciones $y$ hechos encadenados, una serie de elementos que la han llevado a catapultarse, que son de suma importancia precisar.

El factor trascendental, sin duda alguna, es la capacidad gerencial con visión estratégica, así como, los pilares de la estrategia: la acumulación de aliados internos y de alianzas externas, la neutralización de fuerzas opositoras y el involucramiento de la sociedad civil; la presencia de estos en cada fase del proceso de construcción de la estrategia, fue determinante para lograr la situación actual de esta oficina.

Por lo tanto, la estrategia de gestión de la Oficina Municipal de la Mujer del Cantón Central de Heredia, abre un sendero en la institucionalidad pública, al demostrar que la inoperatividad en el Estado puede ser superada por organismos institucionales locales al poner en marcha proyectos que hagan realidad las políticas nacionales y permitan a su población meta hacer efectivos sus derechos.

BIBLIOGRAFÍA

LIBROS

Montgomery, Mariscal. 1969. Historia del arte de la guerra. Madrid: Ed. Aguilar sa.

Paguagua, Estela. 2010. Informe de Labores 2010. Heredia: Oficina Municipal de la Mujer.
Programa Estado de la Nación en Desarrollo Humano Sostenible. 2013. Decimonoveno Informe Estado de la Nación en Desarrollo Humano Sostenible. San José: Programa Estado de la Nación.

Román, Isabel y Morales, Natalia. 2007. Politica Nacional para la Igualdad y Equidad de Género (PIEG) 2007-2017. San José: Instituto Nacional de las Mujeres.

Román, Isabel y Morales, Natalia. 2011. Seguimiento y avances de la PIEG (20072011). San José, Costa Rica: Programa Estado de laNación.

Straface, Fernando y Vargas, Jorge. 2008. "Gestión pública". En Democracia Estable ¿Alcanza?: Análisis de Gobernabilidad en Costa Rica: Gestión Pública, editado por Miguel Gutiérrez Saxe y Fernando Straface, 97-121. Washington dc: Banco Interamericano de Desarrollo.

OTROS

Asamblea Legislativa de la República de Costa Rica. 1998. Ley $N^{\circ}$ 7801. Ley del Instituto Nacional de las Mujeres. Publicada en La Gaceta $\mathrm{N}^{\circ} 94.18$ de mayo.

Asamblea Legislativa de la República de Costa Rica. 2008. Ley $N^{\circ}$ 8679. Modificación de varios artículos del Código Municipal, Ley $\mathrm{N}^{\circ} 7794$. Publicada en la Gaceta $\mathrm{N}^{\circ}$ 233. 2 de diciembre.

Asamblea Legislativa de la República de Costa Rica. 2010. Ley $N^{\circ} 7794$. CódigoMunicipal. Publicada en la Gaceta $\mathrm{N}^{\circ} 143.23$ de julio.

Vargas Acuña, Marianela. 2011. "Perfil de los Mecanismos locales para la igualdad y la equidad de género (melieg)". Documento del INAMU. San José: Instituto Nacional de las Mujeres.

\section{ENTREVISTAS}

Vargas Acuña, Marianela, entrevista por González, Septiembre, 2013. Área Ciudadanía Activa, Liderazgo y Gestión Local, INAMU, Los Yoses.

Fecha de ingreso: 12/09/2014 Fecha de aprobación: 06/03/2015 